OPEN ACCESS

Edited by:

Valentina Pucino,

University of Birmingham,

United Kingdom

Reviewed by:

Cheryl Rockwell,

Michigan State University,

United States

Robert Inman,

University of Toronto Mississauga,

Canada

${ }^{*}$ Correspondence:

Eun Jeong Won

Parasite.woni@jnu.ac.kr

Tae-Jong Kim

ktj1562@jnu.ac.kr

${ }^{\dagger}$ These authors have contributed equally to this work and share first authorship

${ }^{\ddagger}$ These authors have contributed equally to this work

Specialty section: This article was submitted to Autoimmune and

Autoinflammatory Disorders,

a section of the journal

Frontiers in Immunology

Received: 08 October 2020

Accepted: 18 January 2021

Published: 25 February 2021

Citation:

Lee YJ, Kim M-J, Jo S, Jin S-H, Park P-R, Park K, Song H-C, Kim J, Kim J-Y, Shim SC, Kim T-H, Hong S-J, Kang H, Kim T-J and Won EJ (2021) Clonorchis sinensis-Derived

Protein Attenuates Inflammation and New Bone Formation in Ankylosing Spondylitis.

Front. Immunol. 12:615369. doi: 10.3389/fimmu.2021.615369

\section{Clonorchis sinensis-Derived Protein Attenuates Inflammation and New Bone Formation in Ankylosing Spondylitis}

\author{
Yu Jeong Lee ${ }^{1 \dagger}$, Moon-Ju Kim ${ }^{1 \dagger}$, Sungsin $\mathrm{Jo}^{2}$, So-Hee $\mathrm{Jin}^{3}$, Pu-Reum Park ${ }^{3}$, \\ Kijeong Park ${ }^{3}$, Ho-Chun Song ${ }^{4}$, Jahae Kim ${ }^{4}$, Ji-Young Kim ${ }^{5}$, Seung Cheol Shim ${ }^{5}$, \\ Tae-Hwan Kim ${ }^{6}$, Sung-Jong Hong ${ }^{7}$, Hyundeok Kang ${ }^{8}$, Tae-Jong Kim ${ }^{3 *}$ \\ and Eun Jeong Won ${ }^{1 \times \neq}$ \\ ${ }^{1}$ Department of Parasitology and Tropical Medicine, Chonnam National University Medical School, Gwangju, South Korea, \\ ${ }^{2}$ Department of Rheumatology, Hanyang University Institute for Rheumatology Research, Seoul, South Korea, ${ }^{3}$ Department \\ of Rheumatology, Chonnam National University Medical School and Hospital, Gwangju, South Korea, ${ }^{4}$ Department of \\ Nuclear Medicine, Chonnam National University Medical School and Hospital, Gwangju, South Korea, ${ }^{5}$ Division of \\ Rheumatology, Daejeon Rheumatoid \& Degenerative Arthritis Center, Chungnam National University Hospital, Daejeon, South \\ Korea, ${ }^{6}$ Department of Rheumatology, Hanyang University Hospital for Rheumatic Diseases, Seoul, South Korea, \\ ${ }^{7}$ Department of Medical Environmental Biology, Chung-Ang University College of Medicine, Seoul, South Korea, \\ ${ }^{8}$ Department of Biomedical Systems Informatics, Brain Korea 21 PLUS Project for Medical Science, Yonsei University \\ College of Medicine, Seoul, South Korea
}

Helminth infections and their components have been shown to have the potential to modulate and attenuate immune responses. The objective of this study was to evaluate the potential protective effects of Clonorchis sinensis-derived protein (CSp) on ankylosing spondylitis (AS). Cytotoxicity of CSp at different doses was assessed by MTS and flow cytometry before performing experiments. Peripheral blood mononuclear cells (PBMCs) and synovial fluid mononuclear cells (SFMCs) were obtained from AS patients. Inflammatory cytokine-producing cells were analyzed using flow cytometry. The levels of INF- $\gamma$, IL-17A, TNF- $\alpha$, and IL-6 were measured by enzyme-linked immunosorbent assay (ELISA). SKG mice were treated with CSp or vehicles. Inflammation and new bone formation were evaluated using immunohistochemistry, positron emission tomography (PET), and micro-computed tomography (CT). Treatment with CSp resulted in no reduced cell viability of PBMCs or SFMCs until $24 \mathrm{~h}$. In experiments culturing PBMCs and SFMCs, the frequencies of IFN- $\gamma$ and IL-17A producing cells were significantly reduced after CSp treatment. In the SKG mouse model, CSp treatment significantly suppressed arthritis, enthesitis, and enteritis. Micro-CT analysis of hind paw revealed reduced new bone formation in CSp-treated mice than in vehicle-treated mice. We provide the first evidence demonstrating that CSp can ameliorate clinical signs and cytokine derangements in AS. In addition, such CSp treatment could reduce the new bone formation of AS.

Keywords: ankylosing spondylitis, Clonorchis sinensis, inflammation, new bone formation, parasite 


\section{INTRODUCTION}

Ankylosing spondylitis (AS) is a sort of inflammatory arthritis that affects axial skeleton, peripheral joints, and certain extra-articular organs, including the eyes, skin, and gut (1-3). The occurrence of AS is insidious and progressive, leading to spinal deformity, loss of ability to work, disability, and quality-of-life deterioration (4). Although the prevalence of AS varies greatly across geographic regions (5), it has increased from 0.04 to $0.09 \%$ in the USA in recent decades (6). Several researchers have explained this increasing tendency partly by hygiene hypothesis (7), supporting an inverse relationship between worm infection and T helper type 1/17 (Th1/17)-based inflammatory disorders such as rheumatoid arthritis, inflammatory bowel disease, type 1 diabetes, and multiple sclerosis. Although the exact mechanism of AS remains largely unclear, Th1/17 pro-inflammatory mediators are strongly implicated in the initiation and progression of this disease (8-10). Treatment with Tumor necrosis factor (TNF) blockers improves physical function, disease activity, and health-related quality-of-life outcomes $(11,12)$, but not all AS patients respond to the medication. Besides, it is controversial currently whether TNF blockers prevent progression to ankylosis $(13,14)$. Thus, a novel therapeutic strategy for AS is needed.

Recently, many attempts have been made to use parasite administration (e.g., ingestion of eggs of the nematode Trichuris suis) as a new modality for treating autoimmune disorders (15, 16). In animal models of rheumatoid arthritis, some helminth species or their proteins were able to reduce the severity of the clinical symptoms (17-22). However, the role of any trematodesinduced proteins in the pathogenesis and disease activity of AS has yet to be reported. Thus, this study aimed to assess the therapeutic potential of Clonorchis sinensis-induced proteins (CSp) for AS. Results of this study will provide a basis for further clinical applications of $C$. sinensis.

\section{MATERIALS AND METHODS}

\section{Human Samples}

All patients satisfied the modified New York (NY) criteria for AS (23). Peripheral blood mononuclear cells (PBMCs) and synovial fluid mononuclear cells (SFMCs) were obtained from patients with active AS. The demographic characteristics of the patients are shown in Table 1. This study was carried out in compliance with the Helsinki Declaration. It was approved by the Ethics Committee. Written informed consent was obtained from all subjects (CNUH-2011-199).

\section{Clonorchis sinensis Crude Antigen Preparation}

Frozen $C$. sinensis adult worms were mixed with $1 \mathrm{ml}$ of homogenation buffer (5 mM EDTA, 1\% NP-40, $0.2 \mathrm{mM}$

\footnotetext{
Abbreviations: AS, Ankylosing spondylitis; CSp, Clonorchis sinensis-derived protein; i.p., intra-peritoneally; CT, computed tomography; NC, negative control; PBMC, peripheral blood mononuclear cell; PC, positive control; PET, Positron emission tomography; SFMC, synovial fluid mononuclear cells; TNF, Tumor necrosis factor.
}

TABLE 1 | Clinical characteristics of ankylosing spondylitis.

\begin{tabular}{lcc}
\hline & $\begin{array}{c}\text { Peripheral blood } \\
\text { mononuclear cells }\end{array}$ & $\begin{array}{c}\text { Synovial fluid } \\
\text { mononuclear cells }\end{array}$ \\
\hline Total number & 6 & 8 \\
Age, mean \pm SD (years) & $24 \pm 9.5$ & $39.1 \pm 13.7$ \\
Male, $\mathrm{n}(\%)$ & $6(100.0)$ & $4(50.0)$ \\
BASDAl, mean \pm SD & $5.4 \pm 4.0$ & $7.6 \pm 2.1$ \\
AS-DAS, mean \pm SD & $3.6 \pm 2.1$ & $6(75.0)$ \\
HLA-B27, $\mathrm{n}(\%)$ & $6(100.0)$ & $4.6 \pm 3.6$ \\
CRP, mean \pm SD (range, mg/dl) & $2.4 \pm 1.6$ & $2(25.0)$ \\
Recent treatments (last three months) & $3(50.0)$ & $5(62.5)$ \\
Naive, $\mathrm{n}(\%)$ & $3(50.0)$ & $4(50.0)$ \\
NSAIDs use, $\mathrm{n}(\%)$ & $1(16.6)$ & $3(37.5)$ \\
Sulfasalazine use, $\mathrm{n}(\%)$ & $0(0.0)$ & \\
TNF-blocker use, $\mathrm{n}(\%)$ & & \\
\hline
\end{tabular}

BASDAl, Bath Ankylosing Spondylitis Disease Activity Index; HLA, Human Leukocyte Antigen; CRP, C-reactive Protein; NSAIDs, Non-steroid inflammatory drugs; TNF, Tumor Necrosis Factor.

PMSF), homogenized, vortexed for $5 \mathrm{~min}$, and centrifuged at $13,000 \mathrm{rpm}$ for $20 \mathrm{~min}$ at $4^{\circ} \mathrm{C}$. After centrifugation, the supernatant was used for protein extraction using Pierce BCA Protein Assay Kit (Thermo Scientific Co., Rockford, IL, USA) according to the manufacturer's guideline.

\section{Cell Viability Assay}

To determine cell proliferation and cytotoxicity, cells were seeded and stimulated with different concentrations of CSp for indicated time durations. Cell viabilities of PBMCs and SFMCs according to CSp treatment were investigated using a Cell Titer 96 AQueous One Solution Reagent (G3580, Promega, USA). Briefly, $100 \mu \mathrm{l}$ RPMI was mixed with MTS solution $(20 \mu \mathrm{l} /$ well $)$ and added to each well. After incubation, absorbance was recorded at the wavelength of $490 \mathrm{~nm}$ with a 96-well microplate reader (Molecular Devices, USA). For each flow cytometry analysis, whole cells were surface stained with antiFixable Viability Dye-eFluor780 (65-0865-14, Invitrogen, USA).

\section{Co-Culture of Human Inflammatory Cells With CSp}

PBMCs and SFMCs were isolated and suspended in a complete medium (RPMI 1640, 2 mM L-glutamine, 100 units/ml of penicillin, and $100 \mu \mathrm{g} / \mathrm{ml}$ of streptomycin) supplemented with $10 \%$ fetal bovine serum (FBS; Gibco BRL, Grand Island, NY, USA), and then seeded into 96-well plates at cell density of $1 \times$ $10^{6}$ cells/well. Cells in a 96-well culture plate were treated with CSp and then were activated with Dynabeads Human TActivator CD3/CD28 (11131D, Gibco, USA) to obtain a bead to cell ratio of $1: 1$. Cells were then incubated in a humidified $\mathrm{CO}_{2}$ incubator at $37^{\circ} \mathrm{C}$ for $24 \mathrm{~h}$. After stimulating with PMA (100 ng/ $\mathrm{ml})$ and ionomycin $(1 \mu \mathrm{M})$ for $4 \mathrm{~h}$, cells were stained with Pacific Blue-conjugated anti-CD4 (300521, BioLegend, USA), and PEconjugated anti-CD45RO (304205, BioLegend, USA). Cells were washed, fixed, permeabilized with Cytofix/Cytoperm buffer, and stained intracellularly with FITC-conjugated anti-IFN- $\gamma$ (552887, BD, USA), APC-conjugated anti-IL-17A (512334, BioLegend, USA) antibodies followed by analysis with FlowJo Software (BD, USA). In ex vivo cultured supernatants from 
PBMCs, INF- $\gamma$, IL-17A, TNF- $\alpha$, and IL-6 were measured using ELISA (88-7316, 88-7176, 88-7346, and 88-7066, Invitrogen, Austria). The OD was recorded by a SpectraMax ${ }^{\circledR}$ M2 (Molecular Devices Corp., USA) set at $450 \mathrm{~nm}$.

\section{Experimental Animal Model and Clinical Score}

SKG mice on a BALB/c background were purchased from Clea Japan (Tokyo, Japan) and bred under a specific pathogen-free facility. These mice were kept in individually ventilated cages and provided with water and standard diet ad libitum. All experiments were approved by the Institutional Animal Care and Use Committee (CNU IACUC-H-2018-35). They were conducted under the Laboratory Animals Welfare Act, Guide for the Care and Use of Laboratory Animals. Female mice were used in this study. Experiments had three groups: negative control ( $n=10$ mice), vehicle group ( $\mathrm{VH}, n=10$ mice), and CSp treatment group ( $n=10$ mice). For both $\mathrm{VH}$ and CSp treatment groups, a suspension of curdlan (Wako, Osaka, Japan) was intraperitoneally (i.p.) administered at $3 \mathrm{mg} / \mathrm{kg}$ to mice aged 11 weeks. CSp treatment group received CSp $(6 \mu \mathrm{g} / 0.2 \mathrm{ml})$ i.p. twice before arthritis induction. The same dosage was then maintained once a week until sacrifice. Control and VH groups received PBS i.p. instead of CSp. At the start of treatment, randomization was performed based on serial number generation. Clinical signs of mice were monitored twice a week and scored by two independent observers. Scores of the affected joints were summed as follows: $0=$ asymptomatic, 1 = slightly swelling of the ankles or toes, $2=$ ankle swelling severely, 3 = ankle severely swelling and toe swelling, and 4 = ankle and toe swelling and twisting). Sixteen points were the highest possible points.

\section{Positron Emission Tomography and Micro- Computed Tomography Analysis}

A day before sacrifice, mice were fasted for $16 \mathrm{~h}$ prior to undergoing PET/micro CT. Briefly, mice were anesthetized followed by an i.v. injection of $18.5 \mathrm{MBq}{ }^{18} \mathrm{~F}-\mathrm{FDG}$ and scanned sequentially, starting at 30 min post-injection using a small animal PET-CT (SEDECAL, SuperArgus PET/CT 4r, Spain) with a detachable animal bed for maintaining animal position. Anesthesia was maintained by inhalation of approximately $1.5 \%$ isoflurane $/ \mathrm{O}_{2}$ for $1 \mathrm{~L} / \mathrm{min}$ for individual scans and $2 \mathrm{~L} / \mathrm{min}$ to obtain mouse hotel scans administered via nose cone. PET images were reconstructed using OSEM3D (ordered subset expectation maximization)/MAP (maximum a posteriori) algorithm. The volume of interest (VOI) with a diameter of 6-mm was drawn at both sides of the hind paws. Maximal and mean standardized uptake values (SUVx) were then measured. The following CT scan parameters were employed: energy/intensity of $40 \mathrm{kV}$, electric current of 500 $\mu \mathrm{A}$, sample time of $40 \mathrm{~ms}$, and resolution of $768 \times 972$ pixels. Before CT scan, QRM-MicroCT-HA phantom (QRM GmbH; Moehrendorg, Germany) was used for calibration. For segmentation of newly formed bone and normal mature bone, segmentation thresholds values of hind paws and caudal vertebrae were used as described in a previous study (24).

\section{Histological Findings}

At the experimental endpoint, specimens of the ankle and gut were obtained from mice and fixed with $10 \%$ formalin for one week. After fixation, specimens were decalcified in $10 \%$ formic acid with shaking at $37^{\circ} \mathrm{C}$ for a week and embedded in paraffin. Paraffin blocks were sectioned at a thickness of $3.5 \mu \mathrm{m}$ and deparaffinized in neo-clear (109843, Merck, USA), hydrated with graded ethanol, and stained with hematoxylin (105174, Merck, USA) and eosin (HT110216, Sigma, USA). All staining procedures followed standard protocols. Two blinded readers performed pathologic scoring for enthesitis (25) and enteritis (26).

\section{Other Methods}

RNA sequencing, additional flow cytometry, immunoblotting procedure, and sodium dodecyl sulphate-polyacrylamide gel electrophoresis (SDS-PAGE) analysis are described in detail in Supplementary Data 1.

\section{Statistical Analysis}

Symptom score data were assessed using two-way ANOVA with time as a dependent variable. Statistical significance of the difference between means was assessed using the KruskalWallis test with Dunn's multiple comparisons, Wilcoxon matched-pairs signed rank test or Mann-Whitney test. All statistical analyses were performed using Prism 5.0 Software (GraphPad Software, San Diego, CA, USA). For all graphs, $P$ value less than 0.05 was considered as significant and marked as follows: ${ }^{\star} P=0.05-0.01 ;{ }^{\star *} P=0.01-0.001$, and ${ }^{\star *} P<0.001$.

\section{RESULTS}

\section{Cell Viability of CSp Treatment}

There was no significant effect on cell viability with up to $150 \mu \mathrm{g} /$ $\mathrm{ml}$ of CSp (Figure 1A). Treatment with CSp up to $24 \mathrm{~h}$ resulted in no reduced cell viability of PBMCs or SFMCs, analyzed by MTS (Figure 1B) and flow cytometry (Figure 1C). However, reduced viabilities were observed with CSp treatment for 48 and 72 h (Supplementary Figure 1A). In RNA sequencing analysis, down-regulation of several genes related to the metabolic pathway was found after CSp stimulation (Supplementary Figures 1B, C), but there was no significance in the genes related to the apoptosis (Supplementary Figure 1D). Expression levels of INF- $\gamma$ and IL-17A in sequence data were also declined according to the presence of CSp (Supplementary Figure 1E).

\section{CSp Suppresses the Pro-Inflammatory Cytokines in Ex Vivo Experiment}

To explore the anti-inflammatory properties of CSp on cytokines in a human setting, PBMCs and SFMCs obtained from patients with active AS were stimulated and cultured ex vivo for $24 \mathrm{~h}$ in the presence or absence of CSp. In PBMCs and SFMCs, frequencies of IFN- $\gamma$ and IL-17A producing cells in both memory CD4 negative and positive $\mathrm{T}$ cells were significantly reduced after treatment with 


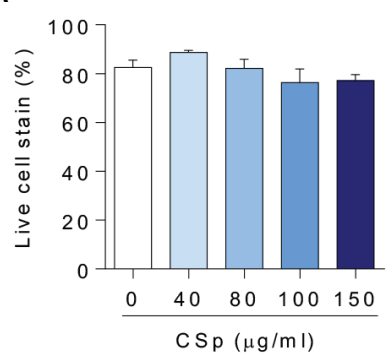

B
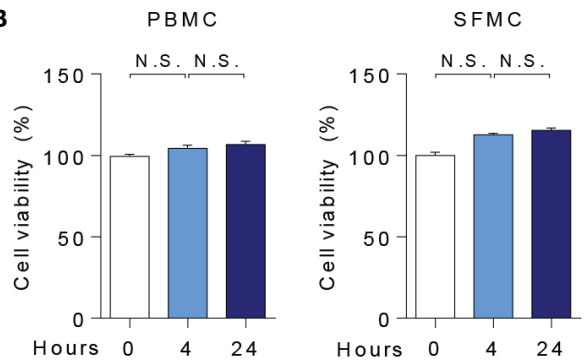

C
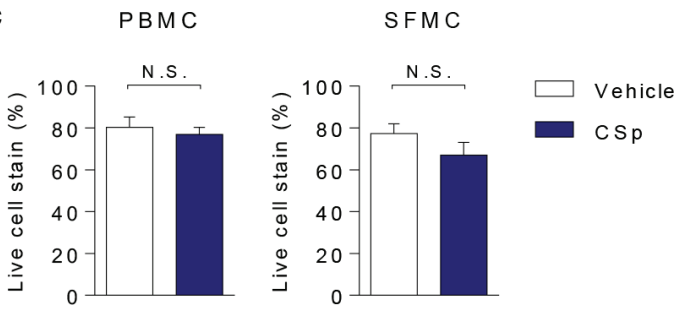

FIGURE 1 | Assessment of cell viability with CSp. (A) Anti-Fixable Viability Dye of PBMCs were analyzed by flow cytometry depending on CSp concentration. Kruskal-Wallis test with Dunn's multiple comparisons was performed to determine statistical significance. Values are the mean \pm SEM (B) Cell viability of PBMCs and SFMCs were analyzed by MTS assay depending on CSp treatment duration. Kruskal-Wallis test with Dunn's multiple comparisons was performed to determine statistical significance. Values are the mean \pm SEM. (C) Viability Dye of PBMCs and SFMCs were stained and measured. Mann-Whitney $U$ test was performed to determine statistical significance. Values are the mean \pm SEM. N.S: not significant. CSp: Clonorchis sinensis-derived protein; PBMCs: peripheral blood mononuclear cell; SFMCs: synovial fluid mononuclear cells.

CSp (Figures 2A, B; Supplementary Figures 2A, B). Dose dependent suppressive effects of IFN- $\gamma$ and IL-17A production in PBMC were observed (Figure 2C). In the supernatant of PBMCs, the production of INF- $\gamma$, IL-17A, TNF- $\alpha$, and IL- 6 were significantly reduced after CSp treatment (Figure 2D). Additionally, further experiments were performed on the effect of CD8 positive T cells or Mucosal-associated invariant T (MAIT) cells in active AS patients. IFN- $\gamma$ producing cells in the CD8 T cells were found to be significantly decreased after CSp treatment (Supplementary Figure 2C). Frequencies of IFN- $\gamma$ and IL-17A producing cells in the MAIT cells were also found to be reduced after CSp treatment (Supplementary Figure 2D). To determine the role of CSp in inflammatory cell signaling, immunoblot assay was performed. We observed that the anti-inflammatory effect of CSp on immune cells was mainly due to the suppression of NF-kB phosphorylation (Supplementary Figure 3).

\section{CSp Treatment Suppresses Clinical Symptoms in SKG Mice}

To investigate the effect of CSp on the development and progression of AS in curdlan-treated SKG mice, mice were treated with either CSp or VH from one week before curdlan injection (Figure 3A). The CSp treatment delayed the onset of arthritis and significantly reduced the severity of arthritis (Figure 3B). At the end of the experiment, CSp injection significantly suppressed the arthritis symptoms $(5.7 \pm 3.68$ vs. $12.8 \pm 2.78, P=0.008)$. Although typical psoriatic skin lesions did not develop, VH-treated mice developed skin redness and hair loss around the ears and nose. However, skin lesions were not obvious in the CSp treatment group (Figure 3C). The frequency of IL-17A producing cells from SKG splenocytes in the CSp treatment group was significantly lower than that from VH-treated mice $(2.74 \pm 0.19 v s .3 .86 \pm 0.39, P=0.024)$. On the other hand, frequencies of IFN- $\gamma$ and TNF- $\alpha$ producing cells were not significantly reduced in the CSp-treated group, compared to those from VH-treated mice $(7.42 \pm 0.64 v s .10 .35 \pm 1.07, P=$ $0.114 ; 6.04 \pm 1.43$ vs. $13.75 \pm 2.84, P=0.087$, respectively) (Supplementary Figure 4). A variety of targets for inflammation imaging have been discovered and utilized. Among them, ${ }^{18}$ F-FDG has been successfully applied in the inflammation realm. ${ }^{18} \mathrm{~F}$-FDGused PET features high sensitivity and specificity. PET has become one of the most frequently used molecular imaging techniques in the clinic (27). Therefore, we used PET images for measuring inflammation on peripheral joints. Representative images are shown in Figure 4A. Mean values of max. SUV of peripheral arthritis from the CSp-treated group were significantly lower than those from VH-treated mice $(1.36 \pm 1.03$ vs. $2.35 \pm 1.15, P=0.0056)$ (Figure 4B). To assess the effect of CSp on local entheseal and gut inflammation, ankle and gut tissues were analyzed histologically. Representative tissue stains at the end of the experiment are shown in Figures 4C, E. Histologic assessment showed that mice treated with CSp had less enthesitis and enteritis than VH-treated mice (enthesitis score, $1.50 \pm 0.57$ vs. $3.55 \pm 0.52, P=0.0047$; enteritis score, $5.17 \pm 0.21$ vs. $8.30 \pm 0.43, P=0.001$ ) (Figures $4 \mathrm{D}, \mathrm{F})$, respectively.

\section{CSp Inhibits New Bone Formation in In Vivo Mice Model}

To evaluate whether CSp could reduce new bone formation, axial and peripheral joints were assessed by micro-CT at the end of treatment. Representative micro-CT images for each group are shown in Figure 5A. Analysis of hind paw revealed less lowdensity bone (used as a measure for new bone) in CSp-treated mice than in VH-treated mice $(5.02 \pm 0.92$ vs. $7.73 \pm 1.91, P=$ 0.001) (Figure 5B). CSp-treated mice exhibited spinal new bone formation similar to $\mathrm{VH}$-treated mice $(57.05 \pm 9.40$ vs. $65.15 \pm$ $10.55, P=0.3383$ ) (Figure 5C). Quantification of normal-density bone $\left(>800 \mathrm{mg}\right.$ of $\mathrm{HA} / \mathrm{cm}^{3}$ ) revealed that mice with CSp treatment had similar bone volume to other groups, suggesting no reduction in normal bone loss (data not shown). 
A
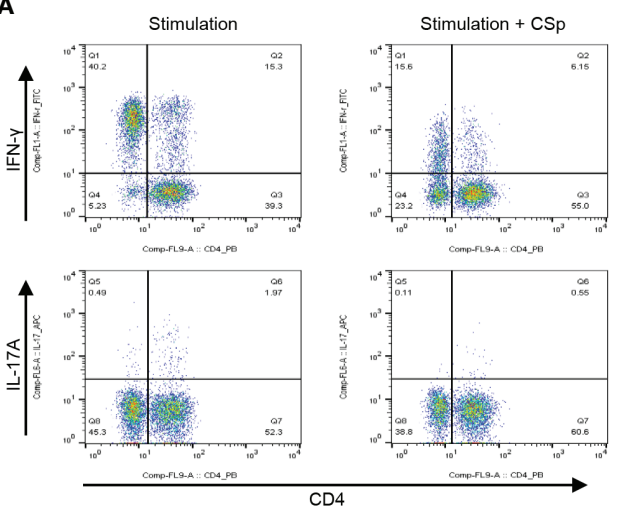

B
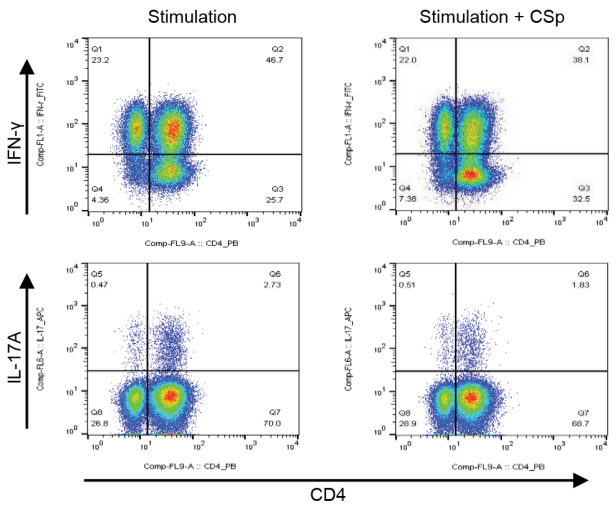

o- Stimulation

- Stimulation + CSp
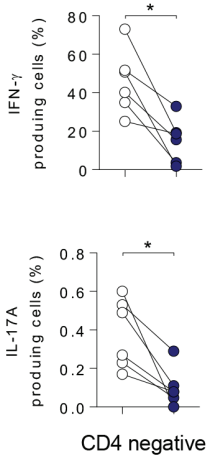

- - Stimulation

- Stimulation + CSp
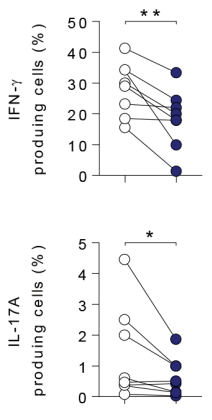

CD4 negative
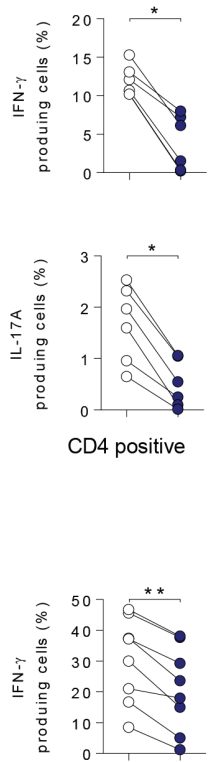

CD4 positive

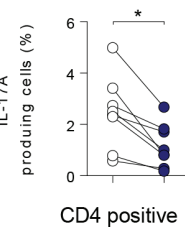

C

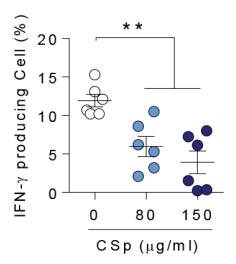

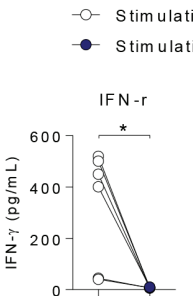

$+\operatorname{csp} T x$

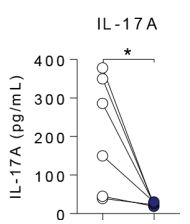

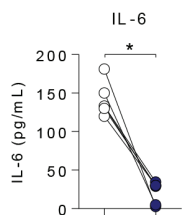

FIGURE 2 | CSp treatment decreases the production of inflammatory cytokines from PBMCs and SFMCs in patients with ankylosing spondylitis. Cells were activated with Dynabeads human CD3/CD28 in the presence or absence of CSp for 24 hours. Then cells were stimulated with PMA and ionomycin for 4 hours. Percentages of INF- $\gamma$ and IL-17A positive cells from PBMCs (A) and SFMC (B) were analyzed. (C) Percentages of INF- $\gamma$ and IL-17A positive cells from PBMCs were analyzed depending on CSp concentration. Kruskal-Wallis test with Dunn's multiple comparisons was performed to determine statistical significance. (D) In ex vivo cultured supernatants from PBMC, INF- $\gamma$, IL-17A, TNF- $\alpha$, and IL-6 were measured by ELISA. Symbols represent the individual sample. ${ }^{*} P<0.05,{ }^{\star \star} P<0.01$, by Wilcoxon matched-pairs signed rank test. CSp: Clonorchis sinensis-derived protein; PBMCs: peripheral blood mononuclear cell; SFMCs: synovial fluid mononuclear cells.

\section{DISCUSSION}

Helminth parasites inhabit immune-competent hosts for a long period of time and appear to develop strategies to induce strong anti-inflammatory responses in the infected host. The inverse prevalence between autoimmune diseases and helminth infection implies a potential protective role of helminth infection in autoimmune diseases. Yet, the effect of any trematode or their products on AS remains unclear. To the best of our knowledge, this is the first study that demonstrates the ameliorative effect of CSp on the clinical signs and cytokine derangements in AS. Moreover, the administration of this CSp could reduce new bone formation in an in vivo AS mice model.

Regarding the helminths therapy targeting arthritis, experimental infections with S. japonicum, S. mansoni, A. suum, and $H$. diminuta, or administration of their proteins have been shown to possess inhibitory effects on murine collagen-induced arthritis (18-22). In these studies, anti-arthritic activity is 
A

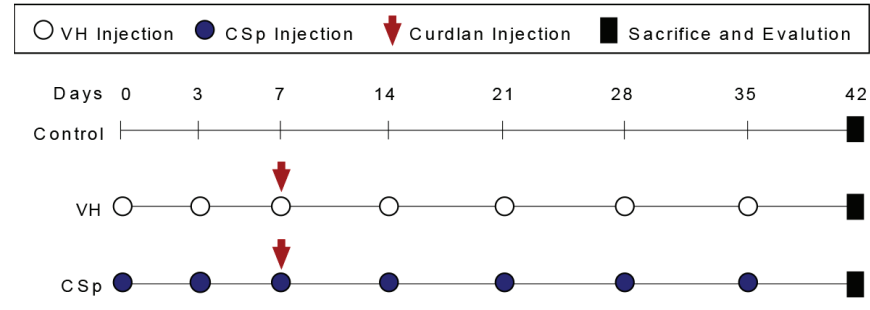

B
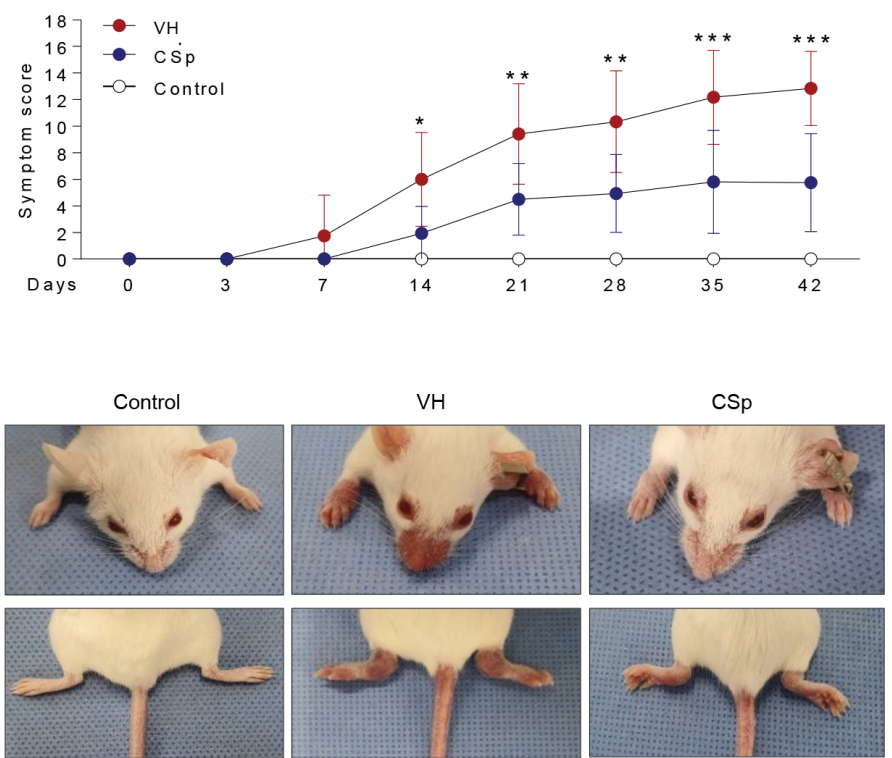

FIGURE 3 | CSp treatment reduces clinical symptoms in SKG mice. (A) SKG mice were treated with CSp or vehicle i.p. for 6 weeks. (B) The arthritis scores were determined based on clinical arthritis severity in each group $\left(n=10\right.$ mice for each group). The values of the left panel are the mean $\pm S D$. ${ }^{*} P<0.05$, ${ }^{* *} P<0.01$, ${ }^{\star \star \star} P<0.001$, by two-way analysis of variance (ANOVA). (C) Representative mouse from each group at the end of the experiment. CSp: Clonorchis sinensis-induced protein; $\mathrm{VH}$ : vehicle group.

mediated by the up-regulation of the Foxp $3^{+}$Tregs with subsequent favorable modulation of both pro- and antiinflammatory cytokines (21). We speculated that $C$. sinensis, a liver fluke found predominantly in Asia including Korea and China, could be an alternative candidate of Schistosoma. Our speculation was supported by previous studies showing an inhibitory effect of $C$. sinensis protein in mice models of asthma and enteritis by inducing $\mathrm{CD} 4^{+} \mathrm{CD} 25^{+} \mathrm{Foxp}^{+} \mathrm{T}$ cells or IL-10 secreting macrophages $(28,29)$.

Up to date, only one study has reported the effect of $C$. sinensis on arthritis (30). In that study, C. sinensis-direct infection has a bad effect on arthritis. It induced an abnormal immune response in mice with collagen-induced arthritis. Although preliminary results from human clinical trials have indicated that treating patients with inflammatory diseases using live helminth parasites has therapeutic potential, the use of helminth products as therapeutic agents might have advantages over a live infection. Thus, we assessed the effect of CSp treatment under suitable conditions excluding poor cell viability. Cell viability was reduced with prolong treatment of CSp. However, according to RNA sequence analysis, it might be related to down regulation of metabolic pathway rather than cell death signals maintaining suppression of IFN- $\gamma$ and IL-17A (Supplementary Figure 1). Notably, this study showed a remarkable decrease of IFN- $\gamma$ and IL-17A production after treatment with CSp in human PBMC and SFMCs, indicating inhibitory effect of CSp on both systemic circulation and regional site. This reduction of inflammatory cytokines was found in both CD4 positive and negative cells without significant changes in the cell proportions (Supplementary Figure 5). In addition, inflammatory cytokines were significantly decreased in CD8 T and MAIT cells after CSp treatment. We found that the antiinflammatory effect of CSp on immune cells was mainly due to the suppression of NF-kB family of transcription factors, which was known to play essential roles in inflammation (31). This phenomenon might serve as the basis of the beneficial effect of 


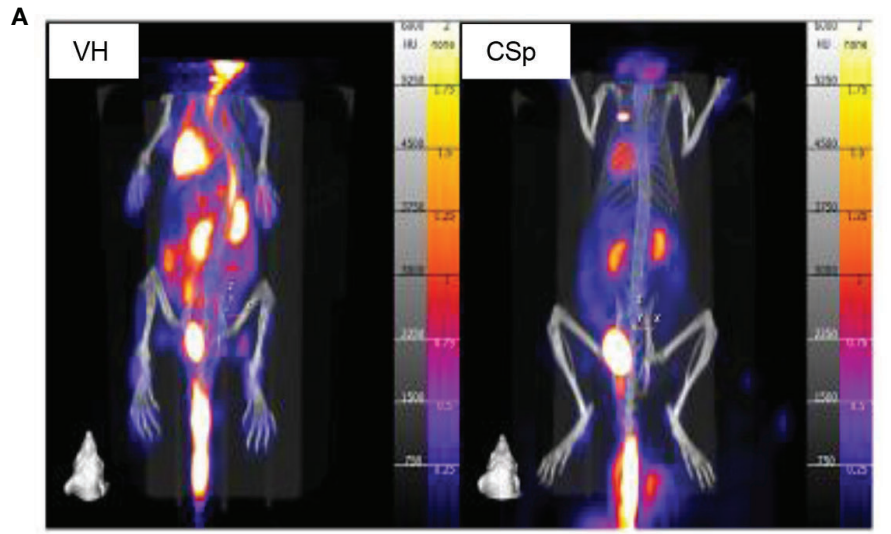

C
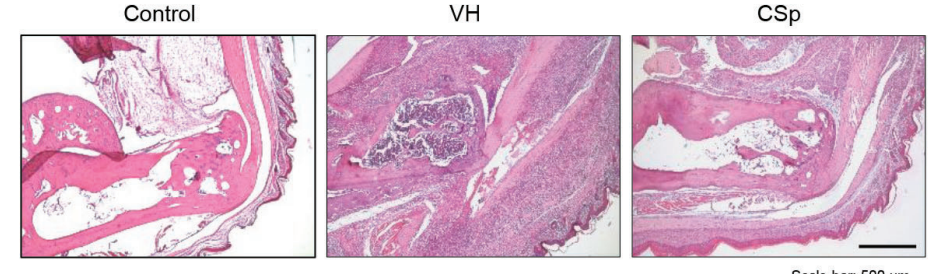

Scale bar: $500 \mu \mathrm{m}$

E
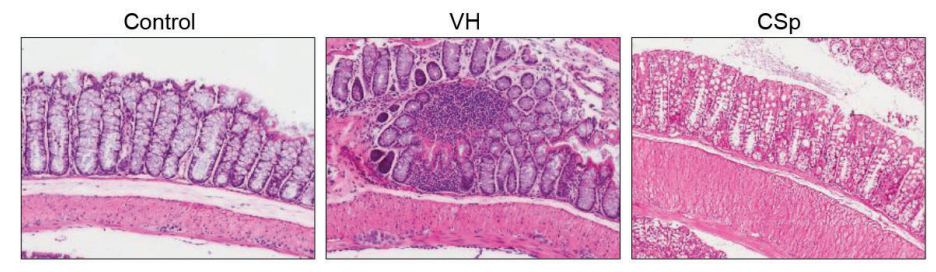

B

PET/CT

(peripheral joints)

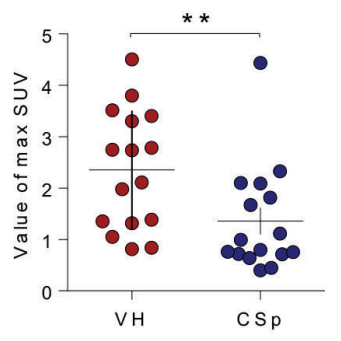

D

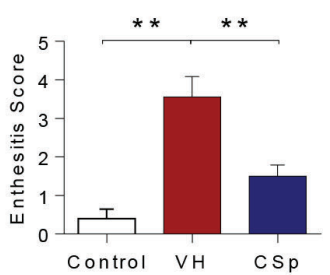

$\mathbf{F}$

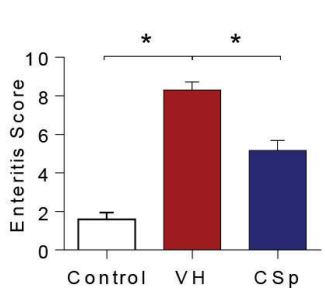

FIGURE 4 | CSp treatment reduces arthritis, enthesitis, and enteritis in SKG mice. Mice were scanned using ${ }^{18} \mathrm{~F}$-FDG PET at the end of the experiment. (A) Representative PET images are shown in each group. (B) The mean values of max. SUV of the peripheral joint were measured. Symbols represent individual ankle joint ( $\mathrm{n}=8$ mice for each group). ${ }^{*} P<0.01$, was determined by Mann Whitney test. (C) Representative heel tissue stains at the end of the experiment are shown. (D) Analysis of histological scores for enthesitis was shown in bar graphs. (E) Representative gut tissue stains at the end of the experiment are shown. (F) Analysis of histological scores for enteritis was shown in bar graphs. Values are the mean $\pm \mathrm{SEM}$. ${ }^{*} P<0.05,{ }^{* *} P<0.01$, by Kruskal-Wallis test with Dunn's multiple comparisons. CSp: Clonorchis sinensis-induced protein; VH: vehicle group.

CSp, thus provides protection against immune-mediated diseases. This finding leads us to clarify the evidence of therapeutic agents against AS using a murine model.

Using SKG mice model, we found that CSp treatment group presented markedly ameliorated disease-specific symptoms and a significantly decreased production of IL-17A in splenocytes. Levels of INF- $\gamma$ and TNF- $\alpha$ seem to decrease in CSp-treated mice compared with PC mice, although the decrease was not statistically significant. PET imaging and histological findings supported the inhibitory effect of CSp treatment on arthritis, enthesitis, and enteritis. In line with our data, similar changes in cells and cytokines such as downregulation of pro-inflammatory cytokines (INF- $\gamma$, TNF- $\alpha$, and IL-17A) have been found in animals infected with S. mansoni (18). Such certain common alterations in the immune response pattern due to parasite might have contributed to the reduced severity of Th1/17-mediated immune disorders including AS. Result of this study suggest that CSp treatment is able to attenuate the symptom severity of AS via systemic and local suppression of pro-inflammatory mediators, further suggesting the potential of therapeutic agents for treating AS.

It is noteworthy that our results suggest that CSp can suppress new bone formation in an in vivo mice model. Effective therapeutic approaches to AS remain a substantial clinical challenge as the suitability of TNF blockade for preventing new bone formation remains controversial. The IL-23/17 axis has been suggested to be a key player in AS pathogenesis and osteoblastogenesis directly $(10,32-34)$. In a recent study, a 

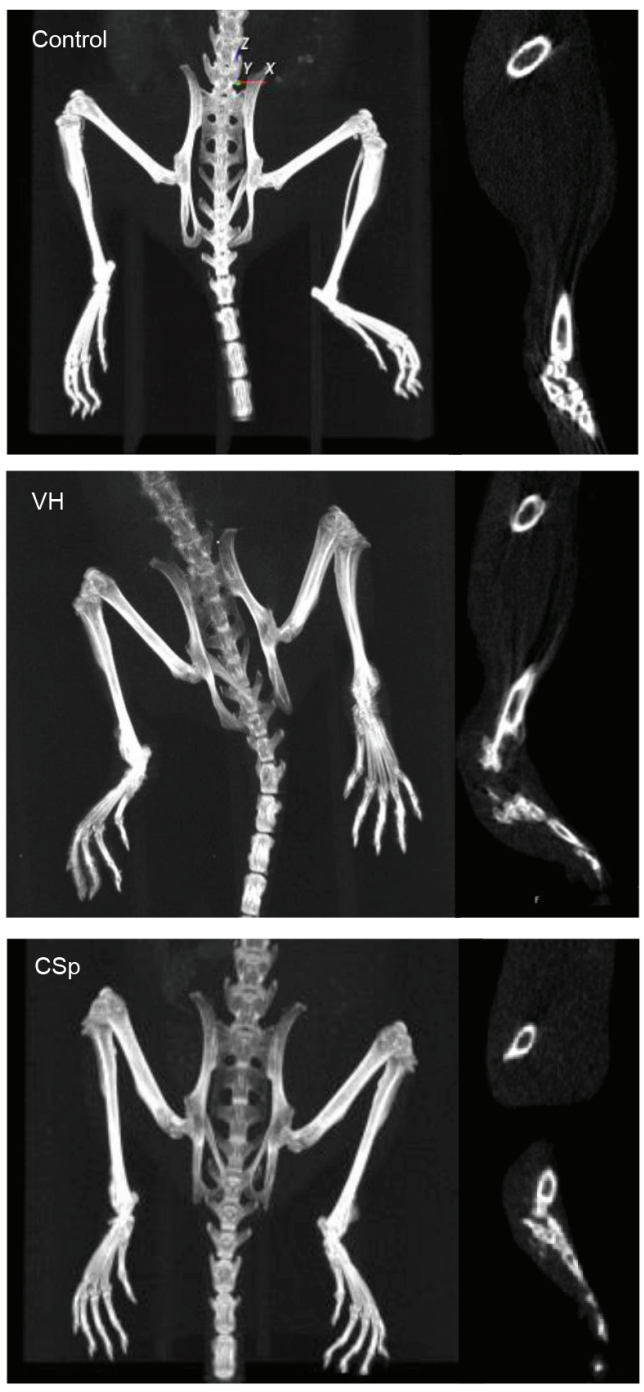

B

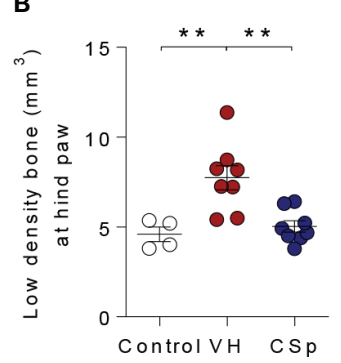

C

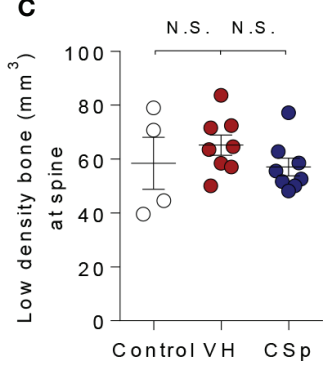

FIGURE 5 | Reduction of new bone formation by CSp in SKG mice model. (A) Representative radiographic images are shown for each group. Quantification of low-density bone (used as a measure for new bone) in hind paws (B) and spine (C) from each group. Symbols represent individual mouse ( $n=4$ mice for control, $n=8$ mice for $V H$ and CSp group). N.S., not significant, ${ }^{\star \star} P<0.01$, by Kruskal-Wallis test with Dunn's multiple comparisons. CSp: Clonorchis sinensis-induced protein; VH: vehicle group. signal transducer and activator of transcription 3 (STAT3) phosphorylation inhibitor was demonstrated to suppress the new bone formation (35). Although the exact mechanism remains unknown, our data highlighted that CSp could be active to inhibit new bone formation in AS which might be accompanied by attenuation of IL-17A via JAK2/STAT3 pathway. CSp did not show significant suppression of spinal new bone formation in this mouse experiment. The current study highlights the further need for elucidating the role of CSp in osteoblastogenesis. Previous studies have shown that it took up to 6 months for observing spinal bony changes in murine models-treated with agents $(36,37)$. Therefore, inadequate timing of anti-osteogenic effect by CSp might be a reason for not inhibiting spinal new bone formation. In contrast to peripheral joint, new bone formation on spines was hardly seen in the images of this study. Therefore, quantitative analysis based on bone density may be an inappropriate tool for measuring spinal bony progression. Because several models mimicking aspects of human spondyloarthritis have been introduced (38), further studies using spinal ankylosis animal models will provide sufficient evidence whether CSp could suppress progression of new bone formation in spine.

This study has several limitations. First, we could not clarify the exact mechanism in the suppression of inflammatory response. However, this might be mediated by the upregulation of regulatory cell populations such as $\mathrm{CD} 4^{+} \mathrm{CD} 25^{+} \mathrm{Foxp}^{+} \mathrm{T}$ cells or IL-10 secreting macrophages $(21,28,29)$. The inverse correlation of IL-10 with Th17 cells has been addressed both in humans and an experimental murine model $(18,39)$. All the findings of these studies support the potential of using CSp to achieve a balance between Th17 cells and Treg cells by altering IL-10 levels as a promising treatment for AS. Second, we have used CSp as crude extracts in the current study. Analysis of CSp by SDS-PAGE revealed many protein bands ranging from 10 to $70 \mathrm{kDa}$ of relative molecular mass (Supplementary Figure 6). Further characterization of the CSp at a molecular level will increase our knowledge of host-parasite interaction that can be applied to standardized therapeutic modality. Third, CSp was administered preventively in the current in vivo study. Therapeutic effect of CSp in the late course of disease should be further assessed on established arthritis.

In summary, our results confirmed that CSp treatment could effectively ameliorate not only inflammation but also new bone formation in AS. Our finding showed that CSp could suppress the pathology associated with AS without compromising the host's ability to fight disease, suggesting that therapies based on the mode of action of CSp might provide novel therapeutic targets for treating AS.

\section{DATA AVAILABILITY STATEMENT}

The original contributions presented in the study are included in the article/Supplementary Material. Further inquiries can be directed to the corresponding authors. 


\section{ETHICS STATEMENT}

The studies involving human participants were reviewed and approved by the Ethics Committee of Chonnam National University Hospital (CNUH-2011-199). The patients/participants provided their written informed consent to participate in this study. The animal study was reviewed and approved by Institutional Animal Care and Use Committee (CNU IACUC-H-2018-35).

\section{AUTHOR CONTRIBUTIONS}

YL, M-JK, EW, and T-JK conceived the study, participated in study design, data analysis, and were responsible for writing and submission of the final manuscript. YL, M-JK, SJ, S-HJ, P-RP, KP, JK, and J-YK carried out the experimental studies. H-CS, SS, and T-HK analyzed and interpreted data. S-JH offered C. sinensis adult worms and interpreted data. HK analyzed RNA-sequence data. All authors contributed to the article and approved the submitted version.

\section{FUNDING}

This study was supported by grants from the National Research Foundation of Korea (NRF) Grant funded by the Ministry of Education, Science, and Technology (grant no. NRF-2019 R1C1C1004605; NRF-2019R1I1A3A01060016; NRF2019M3E5D1A02067953; NRF-2017R1A2B4007994, NRF2019R1A2C2004214), and by the Chonnam National University Hospital Biomedical Research Institute (grant no. BCRI 19047 and BCRI 19048).

\section{ACKNOWLEDGMENTS}

We would like to thank all participants in this study. This manuscript has been released as a pre-print at ResearchSquare, DOI:10.21203/rs.3.rs-72857/v1 (40).

\section{REFERENCES}

1. Exarchou S, Lindstrom U, Askling J, Eriksson JK, Forsblad-d'Elia H, Neovius $\mathrm{M}$, et al. The prevalence of clinically diagnosed ankylosing spondylitis and its clinical manifestations: a nationwide register study. Arthritis Res Ther (2015) 17:118. doi: 10.1186/s13075-015-0627-0

2. Kim TJ, Kim TH. Clinical spectrum of ankylosing spondylitis in Korea. Joint Bone Spine (2010) 77(3):235-40. doi: 10.1016/j.jbspin.2009.11.015

3. Kiltz U, Braun J. Assessments of functioning in patients with axial spondyloarthritis. J Rheum Dis (2020) 27:22-9. doi: 10.4078/jrd.2020.27.1.22

4. Fan D, Liu L, Ding N, Liu S, Hu Y, Cai G, et al. Male sexual dysfunction and ankylosing spondylitis: a systematic review and metaanalysis. J Rheumatol (2015) 42(2):252-7. doi: 10.3899/jrheum.140416

5. Dean LE, Jones GT, MacDonald AG, Downham C, Sturrock RD, Macfarlane GJ. Global prevalence of ankylosing spondylitis. Rheumatol (Oxford) (2014) 53(4):650-7. doi: 10.1093/rheumatology/ket387

6. Walsh J, Hunter T, Schroeder K, Sandoval D, Bolce R. Trends in diagnostic prevalence and treatment patterns of male and female ankylosing spondylitis

\section{SUPPLEMENTARY MATERIAL}

The Supplementary Material for this article can be found online at: https://www.frontiersin.org/articles/10.3389/fimmu.2021. 615369/full\#supplementary-material

Supplementary Figure 1 | Further assessment of cell viability with CSp using MTS assay and RNA sequencing. (A) Cell viability of PBMCs and SFMCs were analyzed by MTS assay depending on duration treated with $150 \mu \mathrm{g} / \mathrm{mL}$ of CSp. (B, C) Gene expression profiles related to the metabolic pathway after CSp treatment. (D) Gene expression profiles related to the apoptosis after CSp treatment. (E) Gene expressions of INF- $\gamma$ and IL-17A by RNA sequencing were shown according to the presence of CSp. CSp: Clonorchis sinensis-derived protein; PBMC: peripheral blood mononuclear cell; SFMCs: synovial fluid mononuclear cell; $\mathrm{VH}$ : vehicle group.

Supplementary Figure 2 | Flow cytometry gating strategies and other T cell response analysis. (A, B) The gating strategies for memory $T$ cells were showen for PBMCs and SFMCs. (C) The production of IFN- $\gamma$ by CD8 T cells among PBMCs were gated and analyzed after CSp stimulation. (D) Inflammatory cytokines from MAIT cells were stained analyzed after CSp stimulation. Symbols represent the individual sample. CSp: Clonorchis sinensis-derived protein; MAIT: Mucosalassociated invariant T; PBMCs: peripheral blood mononuclear cells; SFMCs: synovial fluid mononuclear cells.

Supplementary Figure 3 | Immunoblot analysis of immune cells depending on CSp. Peripheral blood mononuclear cells with or without CSp were activated with PMA and ionomycin for 4 hours. These cells were then lysed for protein extraction and immunoblotting. Representative results of the immunoblot assay are shown.

Supplementary Figure 4 | CSp inhibits IL-17 production on splenocytes in SKG mice. Splenocytes were isolated and stimulated with PMA and ionomycin for 4 hours. Percentages of INF- $\gamma, \mathrm{IL}-17 \mathrm{~A}$ and TNF- $\alpha$ positive cells from total lymphocytes were gated (A) and analyzed (B). Symbols represent the individual sample. ${ }^{*} P<0.05$, by Mann Whitney test. $\mathrm{VH}$ : vehicle; CSp: Clonorchis sinensisderived protein.

Supplementary Figure 5 | CSp does not affect on the proportion of lymphocytes among the peripheral blood mononuclear cells (A) and synovial fluid mononuclear cells (B).

Supplementary Figure 6 | Sodium dodecyl sulphate-polyacrylamide gel electrophoresis (SDS-PAGE) analysis of soluble proteins extracted from C. sinensis. SDS-PAGE showed many protein bands ranging from 10 to $70 \mathrm{kDa}$ of relative molecular mass. Among them, 10-kDa protein was the main molecule in C. sinensis crude extracts. patients in the United States, 2006-2016. BMC Rheumatol (2019) 3:39. doi: 10.1186/s41927-019-0086-3

7. Martinez FD. The coming-of-age of the hygiene hypothesis. Respir Res (2001) 2(3):129-32. doi: $10.1186 / \mathrm{rr} 48$

8. Ranganathan V, Gracey E, Brown MA, Inman RD, Haroon N. Pathogenesis of ankylosing spondylitis - recent advances and future directions. Nat Rev Rheumatol (2017) 13(6):359-67. doi: 10.1038/nrrheum.2017.56

9. Gracey E, Qaiyum Z, Almaghlouth I, Lawson D, Karki S, Avvaru N, et al. IL-7 primes IL-17 in mucosal-associated invariant T (MAIT) cells, which contribute to the Th17-axis in ankylosing spondylitis. Ann Rheum Dis (2016) 75(12):2124-32. doi: 10.1136/annrheumdis-2015-208902

10. Raychaudhuri SP, Raychaudhuri SK. Mechanistic rationales for targeting interleukin-17A in spondyloarthritis. Arthritis Res Ther (2017) 19(1):51. doi: 10.1186/s13075-017-1249-5

11. van der Heijde DM, Revicki DA, Gooch KL, Wong RL, Kupper H, Harnam N, et al. Physical function, disease activity, and health-related quality-of-life outcomes after 3 years of adalimumab treatment in patients with ankylosing spondylitis. Arthritis Res Ther (2009) 11(4):R124. doi: 10.1186/ar2790 
12. Braun J, Baraliakos X, Kiltz U, Kruger K, Burmester GR, Wassenberg S, et al. Disease Activity cutoff values in initiating tumor necrosis factor inhibitor therapy in ankylosing spondylitis: A German GO-NICE Study Subanalysis. J Rheumatol (2020) 47(1):35-41. doi: 10.3899/jrheum.181040

13. Anandarajah A, Ritchlin CT. Treatment update on spondyloarthropathy. Curr Opin Rheumatol (2005) 17(3):247-56. doi: 10.1097/01.bor.0000159926.42761.dd

14. Rudwaleit M, Listing J, Brandt J, Braun J, Sieper J. Prediction of a major clinical response (BASDAI 50) to tumour necrosis factor alpha blockers in ankylosing spondylitis. Ann Rheum Dis (2004) 63(6):665-70. doi: 10.1136/ard.2003.016386

15. Summers RW, Elliott DE, Urban JF Jr., Thompson RA, Weinstock JV. Trichuris suis therapy for active ulcerative colitis: a randomized controlled trial. Gastroenterology (2005) 128(4):825-32. doi: 10.1053/j.gastro.2005.01.005

16. Fleming JO, Isaak A, Lee JE, Luzzio CC, Carrithers MD, Cook TD, et al. Probiotic helminth administration in relapsing-remitting multiple sclerosis: a phase 1 study. Mult Scler (2011) 17(6):743-54. doi: 10.1177/1352458511398054

17. Harnett MM, Melendez AJ, Harnett W. The therapeutic potential of the filarial nematode-derived immunodulator, ES-62 in inflammatory disease. Clin Exp Immunol (2010) 159(3):256-67. doi: 10.1111/j.1365-2249.2009.04064.x

18. Osada Y, Shimizu S, Kumagai T, Yamada S, Kanazawa T. Schistosoma mansoni infection reduces severity of collagen-induced arthritis via downregulation of pro-inflammatory mediators. Int J Parasitol (2009) 39(4):45764. doi: 10.1016/j.ijpara.2008.08.007

19. Song X, Shen J, Wen H, Zhong Z, Luo Q, Chu D, et al. Impact of Schistosoma japonicum infection on collagen-induced arthritis in DBA/1 mice: a murine model of human rheumatoid arthritis. PloS One (2011) 6(8):e23453. doi: 10.1371/journal.pone.0023453

20. Wang X, Li L, Wang J, Dong L, Shu Y, Liang Y, et al. Inhibition of cytokine response to TLR stimulation and alleviation of collagen-induced arthritis in mice by Schistosoma japonicum peptide SJMHE1. J Cell Mol Med (2017) 21 (3):475-86. doi: 10.1111/jcmm.12991

21. Eissa MM, Mostafa DK, Ghazy AA, El Azzouni MZ, Boulos LM, Younis LK. Anti-arthritic activity of Schistosoma mansoni and Trichinella spiralis derivedantigens in adjuvant arthritis in rats: role of FOXP3+ Treg cells. PLoS One (2016) 11(11):e0165916. doi: 10.1371/journal.pone.0165916

22. Shi M, Wang A, Prescott D, Waterhouse CC, Zhang S, McDougall JJ, et al. Infection with an intestinal helminth parasite reduces Freund's complete adjuvant-induced monoarthritis in mice. Arthritis Rheum (2011) 63(2):43444. doi: 10.1002/art.30098

23. van der Linden S, Valkenburg HA, Cats A. Evaluation of diagnostic criteria for ankylosing spondylitis. A proposal for modification of the New York criteria. Arthritis Rheum (1984) 27(4):361-8. doi: 10.1002/art.1780270401

24. van Tok MN, van Duivenvoorde LM, Kramer I, Ingold P, Pfister S, Roth L, et al. Interleukin-17A inhibition diminishes inflammation and new bone formation in experimental spondyloarthritis. Arthritis Rheumatol (2019) 71 (4):612-25. doi: 10.1002/art.40770

25. Benham H, Rehaume LM, Hasnain SZ, Velasco J, Baillet AC, Ruutu M, et al. Interleukin-23 mediates the intestinal response to microbial beta-1,3-glucan and the development of spondyloarthritis pathology in SKG mice. Arthritis Rheumatol (2014) 66(7):1755-67. doi: 10.1002/art.38638

26. Gracey E, Hromadova D, Lim M, Qaiyum Z, Zeng M, Yao Y, et al. TYK2 inhibition reduces type 3 immunity and modifies disease progression in murine spondyloarthritis. J Clin Invest (2020) 130(4):1863-78. doi: 10.1172/ JCI126567

27. Wu C, Li F, Niu G, Chen X. PET imaging of inflammation biomarkers. Theranostics (2013) 3(7):448-66. doi: 10.7150/thno.6592
28. Jeong YI, Kim SH, Ju JW, Cho SH, Lee WJ, Park JW, et al. Clonorchis sinensisderived total protein attenuates airway inflammation in murine asthma model by inducing regulatory $\mathrm{T}$ cells and modulating dendritic cell functions. Biochem Biophys Res Commun (2011) 407(4):793-800. doi: 10.1016/j.bbrc.2011.03.102

29. Jang SW, Cho MK, Park MK, Kang SA, Na BK, Ahn SC, et al. Parasitic helminth cystatin inhibits DSS-induced intestinal inflammation via IL-10(+) F4/80(+) macrophage recruitment. Korean J Parasitol (2011) 49(3):245-54. doi: 10.3347/kjp.2011.49.3.245

30. Li X, Yang Y, Qin S, Kong F, Yan C, Cheng W, et al. The impact of Clonorchis sinensis infection on immune response in mice with type II collagen-induced arthritis. BMC Immunol (2020) 21(1):7. doi: 10.1186/s12865-020-0336-6

31. Hoesel B, Schmid JA. The complexity of NF-kappaB signaling in inflammation and cancer. Mol Cancer (2013) 12:86. doi: 10.1186/1476-4598-12-86

32. Kenna TJ, Davidson SI, Duan R, Bradbury LA, McFarlane J, Smith M, et al. Enrichment of circulating interleukin-17-secreting interleukin-23 receptorpositive gamma/delta $\mathrm{T}$ cells in patients with active ankylosing spondylitis. Arthritis Rheum (2012) 64(5):1420-9. doi: 10.1002/art.33507

33. Lubberts E. The IL-23-IL-17 axis in inflammatory arthritis. Nat Rev Rheumatol (2015) 11(7):415-29. doi: 10.1038/nrrheum.2015.53

34. Jo S, Wang SE, Lee YL, Kang S, Lee B, Han J, et al. IL-17A induces osteoblast differentiation by activating JAK2/STAT3 in ankylosing spondylitis. Arthritis Res Ther (2018) 20(1):115. doi: 10.1186/s13075-018-1582-3

35. Jo S, Won EJ, Kim MJ, Lee YJ, Jin SH, Park PR, et al. STAT3 phosphorylation inhibition for treating inflammation and new bone formation in ankylosing spondylitis. Rheumatol (Oxford) (2020). doi: 10.1093/rheumatology/keaa846

36. Jo S, Lee EJ, Nam B, Kang J, Lee S, Youn J, et al. Effects of dihydrotestosterone on osteoblast activity in curdlan-administered SKG mice and osteoprogenitor cells in patients with ankylosing spondylitis. Arthritis Res Ther (2020) 22 (1):121. doi: 10.1186/s13075-020-02217-9

37. Li X, Wang J, Zhan Z, Li S, Zheng Z, Wang T, et al. Inflammation intensitydependent expression of osteoinductive Wnt proteins is vritical for rctopic new bone gormation in ankylosing spondylitis. Arthritis Rheumatol (2018) 70 (7):1056-70. doi: 10.1002/art.40468

38. Vieira-Sousa E, van Duivenvoorde LM, Fonseca JE, Lories RJ, Baeten DL. Review: animal models as a tool to dissect pivotal pathways driving spondyloarthritis. Arthritis Rheumatol (2015) 67(11):2813-27. doi: 10.1002/art.39282

39. Heo YJ, Joo YB, Oh HJ, Park MK, Heo YM, Cho ML, et al. IL-10 suppresses Th17 cells and promotes regulatory $\mathrm{T}$ cells in the CD4+ $\mathrm{T}$ cell population of rheumatoid arthritis patients. Immunol Lett (2010) 127(2):150-6. doi: 10.1016/j.imlet.2009.10.006

40. Lee YJ, Kim MJ, Jo S, Jin SH, Park PR, Song HC, et al. Clonorchis sinensisderived protein attenuates inflammation and new bone formation in ankylosing spondylitis. Arthritis Res Ther (2020), ARRT-D-20-00726.

Conflict of Interest: The authors declare that the research was conducted in the absence of any commercial or financial relationships that could be construed as a potential conflict of interest.

Copyright (c) 2021 Lee, Kim, Jo, Jin, Park, Park, Song, Kim, Kim, Shim, Kim, Hong, Kang, Kim and Won. This is an open-access article distributed under the terms of the Creative Commons Attribution License (CC BY). The use, distribution or reproduction in other forums is permitted, provided the original author(s) and the copyright owner(s) are credited and that the original publication in this journal is cited, in accordance with accepted academic practice. No use, distribution or reproduction is permitted which does not comply with these terms. 